\title{
PROPOSIÇÃO DE UM MODELO CRUSTAL PARA A REGIÃO DO CONE DO AMAZONAS BASEADO EM DADOS GRAVIMÉTRICOS
}

\author{
Luiz Gabriel Souza de Oliveira, ${ }^{1}$ Marcelo Augusto Martins Neto, ${ }^{2}$ Maria Sílvia Carvalho Barbosa ${ }^{3}$ \\ Recebido em 4 abr., 2003 / Aceito em 19 jan., 2004 \\ Received Apr. 4, 2003 / Accepted Jan. 19, 2004
}

\begin{abstract}
The Amazon Cone, located in the Brazilian Equatorial Margin, is constituted by a large deltaic sedimentation, which started in the Middle Miocene in response to the reversion of the Amazon river drainage following the main Andean orogenic stage. The crustal arrangement in the Amazon Cone region was investigated in this study, based on an integrated analysis of gravity data. Crustal configuration showed by 2-D gravity modelling based on density models constructed with seismic interpretation results, allowing to verify: (1) sedimentary thickness; (2) crustal thickness and (3) morphology and depth of the crust-mantle interface. Non-linear inversion algorithm was used, applying a density model with exponential variation (in terms of depth). Thus, depths of the crust-mantle interface and crustal thickness values were adjusted, being coherent with refraction seismic data. The reached results in this work allowed the characterization of the crustal structure in the region, as well as the observation of the principal geological features.
\end{abstract}

Keywords: gravity, 2-D gravity modelling, non-linear inversion, crustal structure.

\section{RESUMO}

O Cone do Amazonas, situado na Bacia da Foz do Amazonas, Margem Equatorial Brasileira, é caracterizado por um espesso pacote sedimentar terrígeno depositado a partir do Mioceno Médio, como consequiência da reversão da drenagem do rio Amazonas decorrente da orogenia Andina. Este trabalho investigou, por meio de uma análise integrada de dados gravimétricos, a arquitetura crustal da região do Cone do Amazonas. Modelagens gravimétricas 2D, baseadas em modelos de densidades construídos a partir de informações sísmicas, mostraram a espessura sedimentar, a espessura crustal, a morfologia e a profundidade da interface crostamanto. Aplicou-se um algoritmo de inversão não-linear, adotando-se um modelo de densidades variando exponencialmente com a profundidade para a crosta. Assim, os valores de profundidade da interface crosta-manto, bem como de espessura crustal, foram ajustados, tornando-se compatíveis com valores obtidos por sísmica de refração. Os resultados alcançados neste estudo permitiram a caracterização da crosta na região do Cone do Amazonas, possibilitando a observação das principais características de sua estruturação geológica.

Palavras-chave: Gravimetria, Modelagem Gravimétrica 2D, Inversão não-linear, Estrutura Crustal.

1 Departamento de Física - Instituto de Ciências Exatas e Biológicas/Universidade Federal de Ouro Preto. Doutorando PPG-ECRN - Departamento de Geologia/Escola de Minas/Universidade Federal de Ouro Preto, End: Campus Morro do Cruzeiro s/n , CEP 35400-000 0uro Preto - Minas Gerais. Tel: (31)3559-1605. E-mail: luizgabriel@degeo.ufop.br

2 PPG-ECRN - Departamento de Geologia/Escola de Minas/Universidade Federal de Ouro Preto, NUPETRO - Núcleo de Geologia do Petróleo / Fundação Gorceix, End: Campus Morro do Cruzeiro s/n, CEP 35400-000 0uro Preto - Minas Gerais. Tel: (31)3559-1605. E-mail: mamneto@uai.com.br.

3 PPG-ECRN - Departamento de Geologia/Escola de Minas/Universidade Federal de Ouro Preto, End: Campus Morro do Cruzeiro s/n, CEP $35400-000$ 0uro Preto - Minas Gerais. Tel: (31)35591605. E-mail: silvia@degeo.com.br 


\section{INTRODUÇÃOO}

0 desenvolvimento significativo da geofísica nas duas últimas décadas, decorrente do avanço tecnológico e do surgimento de novas técnicas de coleta, processamento e interpretação de dados, foi fundamental para a evolução do conhecimento geotectônico das bacias sedimentares.

Perante esse contexto, os métodos potenciais constituem ferramentas eficientes no estudo da estruturação, mecanismos condicionantes e evolução tectônica de bacias sedimentares.

Sendo assim, a gravimetria proporciona 0 conhecimento das estruturas geológicas, além de fornecer informações relevantes sobre 0 comportamento do embasamento cristalino, da espessura crustal e do equilibrio isostático (SHARMA, 1986).

0 presente artigo tem por objetivo propor um modelo crustal para a região do Cone do Amazonas, Bacia Foz do Amazonas, baseado na modelagem e inversão de dados gravimétricos obtidos por levantamento marinho. Constituem também produtos deste trabalho a determinação da espessura crustal, bem como a profundidade da interface crosta-manto na região.

0 Cone do Amazonas já foi alvo de trabalhos baseados na interpretação de dados gravimétricos (COCHRAN, 1973; BRAGA, 1991; MENDONÇA; EL-ROBRINI, 1993; OLIVEIRA, 2003), visando tanto 0 entendimento do seu arcabouço estrutural quanto de sua evolução tectônica, além da investigação das propriedades mecânicas da litosfera na regiáo.

\section{GEOLOGIA DA ÁREA DE ESTUDO}

0 Cone do Amazonas situa-se na margem continental norte brasileira (Figura 1), a noroeste da atual foz do Rio Amazonas (DAMUTH; KUMAR, 1975), ocupando uma área total de $120 \mathrm{mil} \mathrm{km}^{2}$, que corresponde a cerca de 45\% da área da Bacia da Foz do Amazonas.

Damuth; Flood (1984) dividem o Cone do Amazonas em três regiões distintas, segundo características morfológicas: Cones superior, médio e inferior.

Devido ao soerguimento dos Andes no Mioceno Médio, a drenagem do rio Amazonas sofreu uma inversão no seu fluxo, passando a desaguar no Oceano Atlântico. Coincidentemente, uma regressão marinha permitiu a chegada de grandes quantidades de sedimentos terrígenos além da quebra da plataforma, iniciando a formação do Cone do Amazonas, que evoluiu segundo períodos regressivos a partir do Mioceno Médio (ARAÚJJ; THELLEN, 1993).

A estratigrafia do Cone do Amazonas é representada por uma sequiência deposicional datada do Mioceno Médio ao Recente (BRANDÃO; FEIJÓ, 1994), composta por areias grossas de origem fluvial (Formação

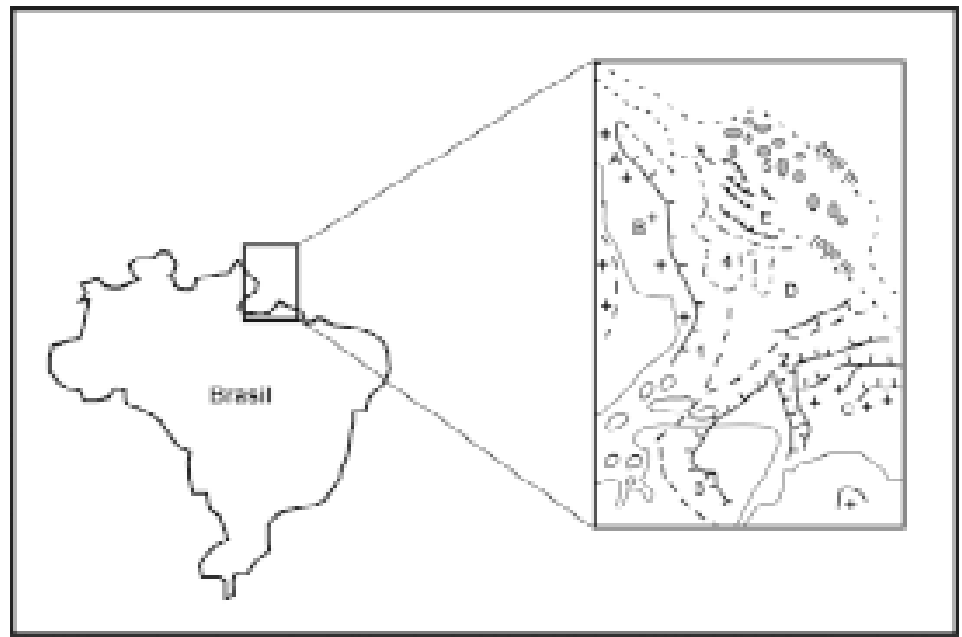

Figura 1 - Mapa esquemático apresentando a localização e e arcabouço estrưưral da Bacia da Foz do Amazonas (OLIVEIRA, 2003). Legenda: A - Subbacia Caciporé; B - Plataforma do Amapá; C - Plataforma Ilha de Santana; D - Plataforma Carbonática;

E - Cone do Amazonas; 1 - Graben Mexiana; 2 - Graben Mexiana Oriental; 3 - Graben Limoeiro; 4 - Calha Oligocênica; 5 - Diápiros de argila.

Figure 1 - Tectonic elements and regional setting of the Foz do Amazonas basin (OLIVEIRA, 2003).

Legend: A - Caciporé sub-basin; B - Amapá shelf; C - Ilha de Santana shelf; D - Carbonatic shelf;

E - Amazon cone; 1 - Mexiana graben; 2 - Eastern Mexiana graben; 3 - Limoeiro graben; 4 - Oligocene channel; 5 - Diapirs. 
Tucunaré), arenitos finos, siltitos e argilitos (Formação Pirarucu) e por folhelhos e argilitos de talude (Formação Orange).

Em relação ao seu padrão estrutural, fori encontrado, através do estudo de seções sísmicas de reflexão no Cone (OLIVEIRA, 2003), um complexo sistema de falhas de crescimento - de natureza lístrica, associado a um domínio de falhas de empurrão, além de expressivos diápiros de argila.

\section{DADOS DISPONÍVEIS}

Os dados gravimétricos utilizados neste estudo (Figura 2) provêm do Mapa de Anomalias Bouguer da Margem Continental Amazônica (OLIVEIRA, 2003).

A construção daquele mapa foi baseada nos dados provenientes do levantamento realizado pelo Projeto EQUANT II (NESS et al., 1989),

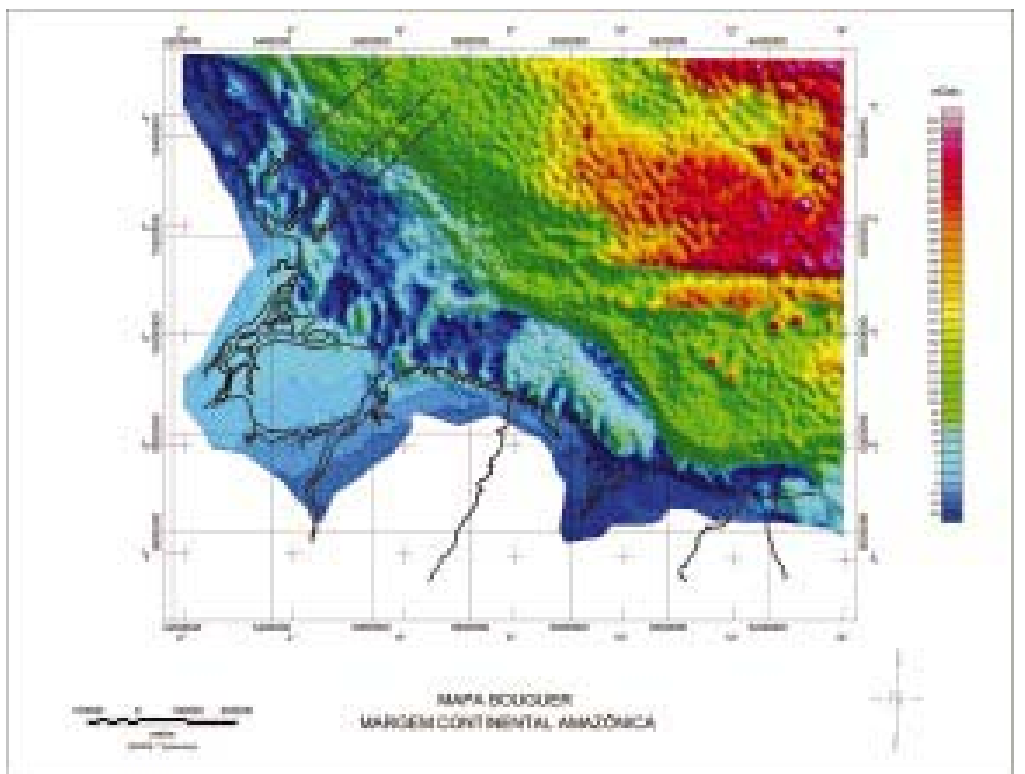

Figura 2 - Mapa de anomalias Bouguer da Margem Continental Amazônica, com a localização dos perfis gravimétricos utilizados neste estudo Figure 2 - Bouguer anomaly map of the Amazon Continental Margin and location of the profiles used in this study

planejado e executado pela Petrobras em parceria com a Oregon State University, tendo como objetivo precípuo a coleta de dados batimétricos, gravimétricos e magnetométricos.

0 recobrimento do levantamento estendeu-se por $20.700 \mathrm{~km}$, entre São Luís e a borda da Guiana Francesa. 0 sistema de navegação utilizado foi o ARGO. Os valores de aceleração da gravidade foram obtidos através de um gravímetro digital LaCoste \& Romberg S-42, de registro contínuo, acoplado a uma plataforma estabilizadora e com o período de oscilação de três minutos. A base gravimétrica esteve localizada em Belém.

0 processamento das informaç̃es levantadas seguiu os passos básicos da operação de redução dos dados gravimétricos: correções de latitude, driftinstrumental, Eötvos, Ar-livre e Bouguer.

\section{MODELAGEM GRAVIMÉTRICA 2D}

\section{Fundamentos}

Segundo 0 clássico trabalho de Talwani, Worzel e Landisman (1959), corpos e estruturas geológicas podem ter suas feições aproximadas por um conjunto de polígonos fechados, onde é possível calcular, por meio de expressões analíiticas, as componentes horizontal e vertical da atração gravitacional que estes exercem sobre um ponto qualquer (Figura 3).

Portanto, a componente vertical da atração gravitacional $(V)$ de um polígono de $n$ lados e de densidade sé calculada pela seguinte equação: 


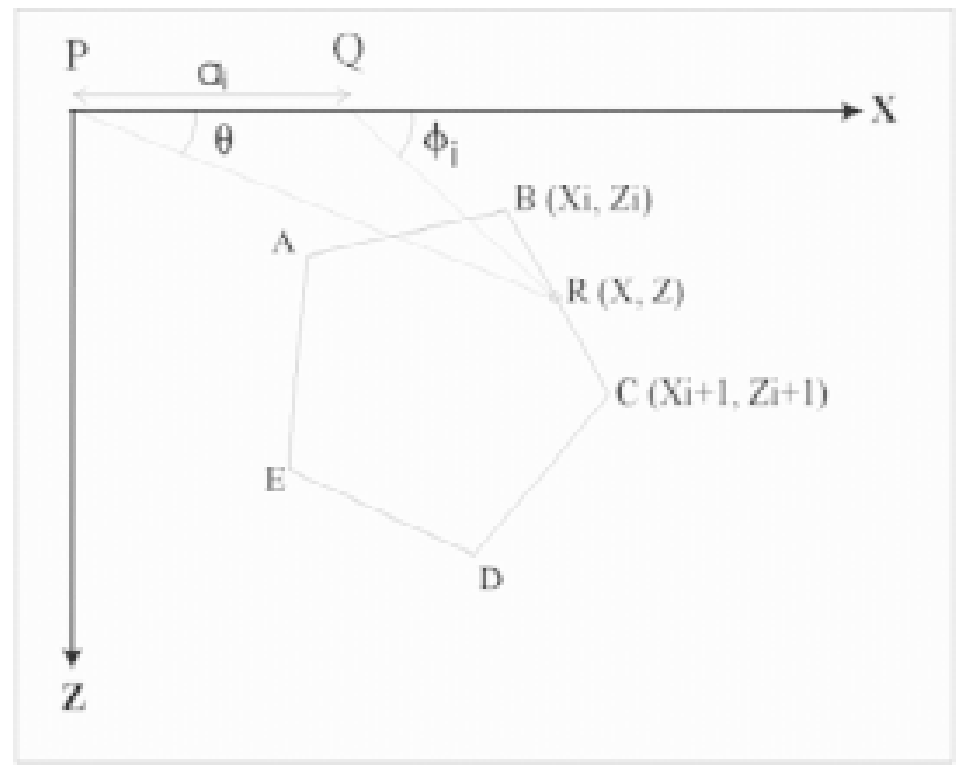

Figura 3 - Elementos geométricos envolvidos na atração gravitacional gerada por um polígono (TALWANI; WORZEL; LANDISMAN, 1959) Figure 3 - Geometric elements involved in the gravity attraction created by a polvgon (TALWANI; WORZEL; LANDISMAN, 1959)

$V=2 G \sigma \sum_{i=l}^{n} Z_{1}$

onde $G$ é a constante universal da gravitação e $Z_{i}$ é dada pela relação:

$\mathrm{Z}_{1}=\mathrm{a}_{\mathrm{i}} \operatorname{sen} \phi, \cos \theta_{\mathrm{i}}\left[\theta_{1}-\theta_{\mathrm{i}+1}+\tan \phi_{\mathrm{i}} \ln \frac{\cos \theta_{\mathrm{i}}\left(\tan \phi_{\mathrm{i}}-\tan \phi_{\mathrm{i}}\right)}{\cos \theta_{\mathrm{i}+1}\left(\tan \phi_{\mathrm{i}+1}-\tan \phi_{\mathrm{i}}\right)}\right]$

com

$$
\begin{aligned}
& \theta_{i}=\arctan \frac{z_{i}}{x_{i}} \\
& \theta_{i}=\arctan \frac{z_{i+1}-z_{i}}{x_{i+1}-x_{i}} \\
& \theta_{i+1}=\arctan \frac{z_{i+1}}{x_{i+1}} \\
& a=x_{i+1}+z_{i+1} \frac{x_{i+1}-x_{i}}{z_{i}-z_{i+1}}
\end{aligned}
$$

\section{METODOLOGIA PARA CONSTRUÇÃO DOS MODELOS CRUSTAIS}

A construção dos modelos crustais bidimensionais baseou-se nos modelos de densidades oriundos da interpretação de dados de sísmica de reflexão. Este procedimento procurou reduzir o caráter ambíguo dos modelos gravimétricos (WHAR, 1996), e, portanto, postergou maior credibilidade aos modelos crustais propostos.

Estes modelos são compostos pelos seguintes polígonos: manto superior, crosta continental, crosta transicional, crosta oceânica, seqüência rifte (Jurássico-Cretáceo Inferior), seqüência marinha 1 (Cretáceo Superior-Mioceno Médio) e seqüência marinha 2 (Mioceno MédioHoloceno), com valores de densidades listados na Tabela 1. Maiores

TABELA 1 - Valores de densidades utilizadas na modelagem gravimétrica 2D. Dados compilados de Sperle, Mello e Costa (1989) e Braga (1991) TABLE I - Densities applied in the 2D-gravity modelling, based in Sperle, Mello e Costa (1989) and Braga (1991)

\begin{tabular}{|c|c|}
\hline Horizonte & Densidade $\mathbf{( g / \mathbf { c m } ^ { 3 } )}$ \\
\hline água & 1.03 \\
\hline diápiros de argila & 2.1 \\
\hline seqüência marinha 2 & 2.4 \\
\hline seqüência marinha 1 & 2.55 \\
\hline seqüência rifte & 2.65 \\
\hline crosta continental & 2.8 \\
\hline crosta transicional & 2.82 \\
\hline crosta oceânica & 2.85 \\
\hline manto superior & 3.25 \\
\hline
\end{tabular}




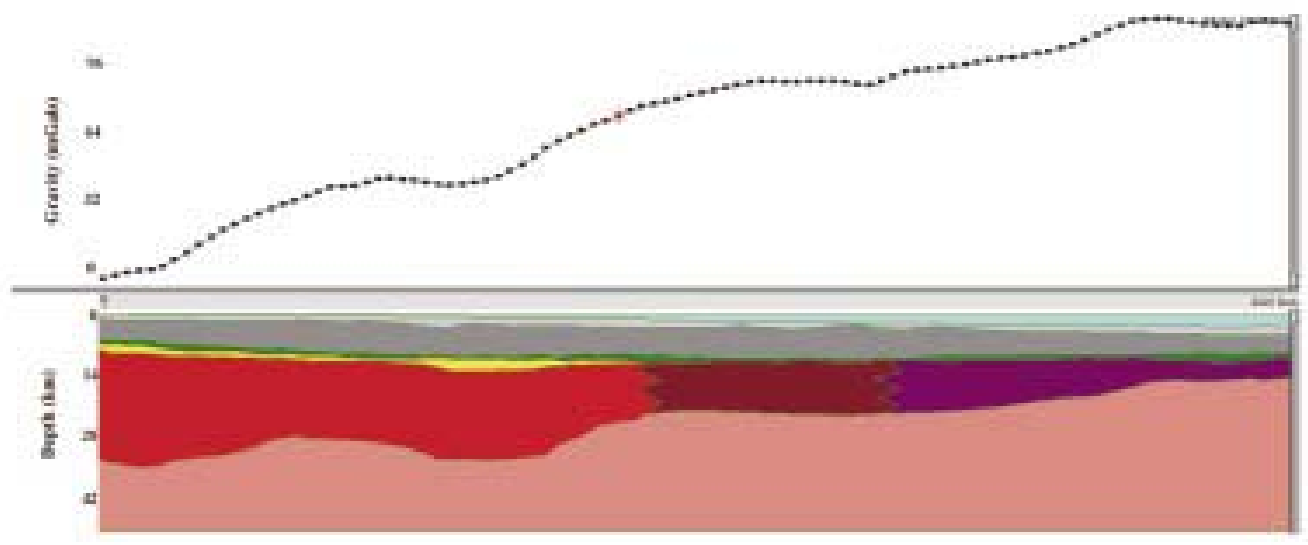

(a)

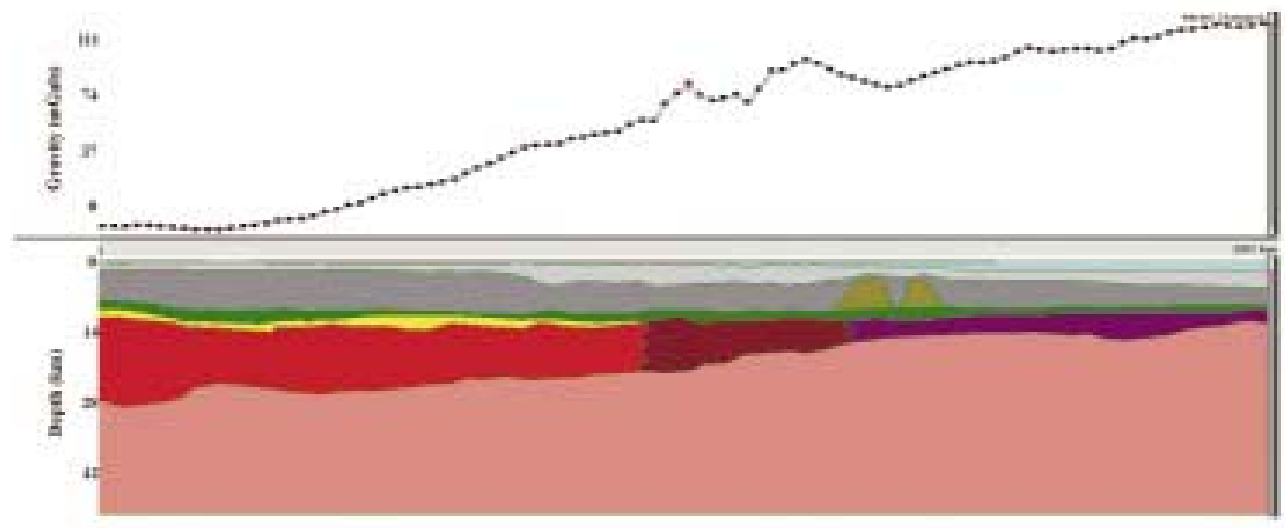

(b)

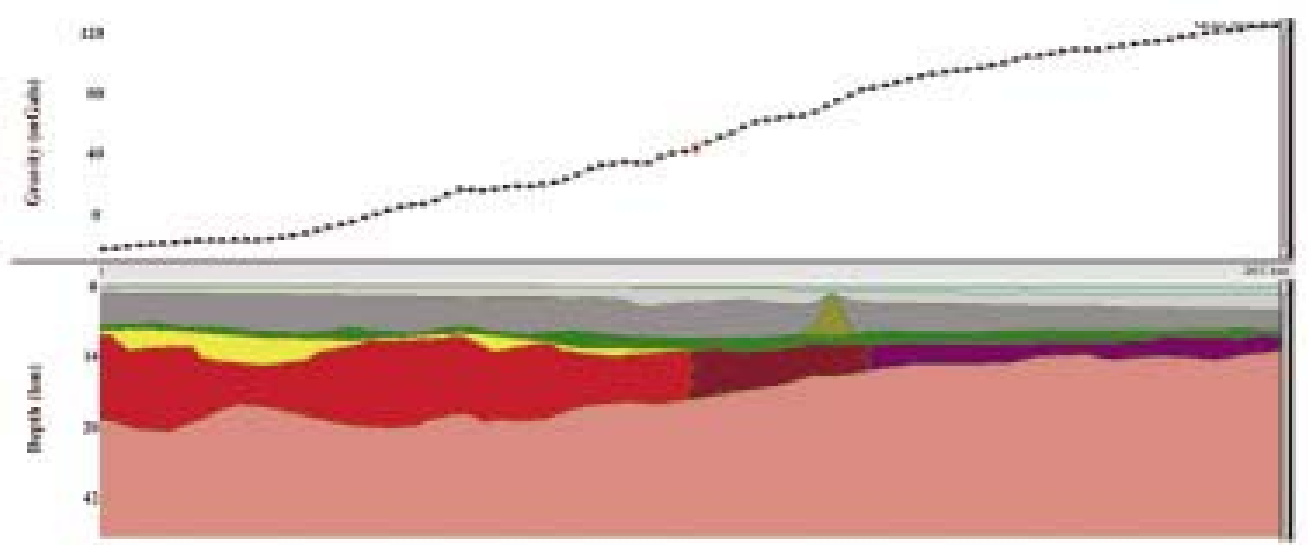

(c)

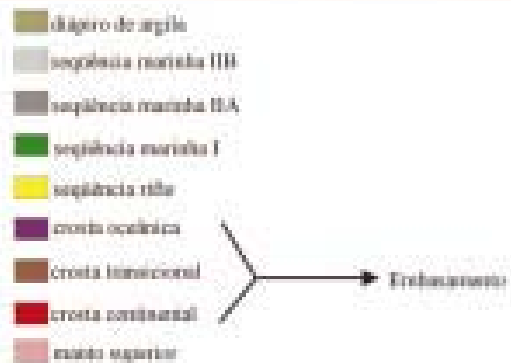

Figura 4 - Modelos crustais obtidos pela modelagem gravimétrica 2D: a) GRAV01; b) GRAV02; c) GRAV03 Figure 4 - (rustal models obtained by 2D-gravity modelling: a) GRAVOI; b) GRAVO2; c) GRAVO3 
detalhes sobre a interpretação dos dados sísmicos e a geração dos modelos de densidades podem ser encontrados em Oliveira (2003).

Algumas convenções foram adotadas na realização da modelagem 2D: a) adoção de valores de densidade constantes; b) expansão lateral do modelo, com a intenção de evitar 0 efeito de borda nos resultados obtidos; c) presença de uma crosta transicional nos modelos.

Procurou-se atingir um melhor ajuste entre as anomalias observadas e as calculadas a partir do modelo. Os resultados estão disponíveis na Figura 4.

Algumas observações foram feitas a partir da análise dos resultados: a) maior espessura crustal na região onshore, com diminuição gradual no sentido offshore, b) geometria da interface crosta-manto bem caracterizada pelos perfis Bouguer; c) estruturação do embasamento em grabens e horsts bem destacadas nos modelos GRAV02 e GRAV03; d) presença de diápiros de argila nos perfis GRAV02 e GRAV03, que também são visíveis nos dados sísmicos.

Com base nos resultados, elaborou-se mapas de profundidade da interface crosta-manto, espessura sedimentar e espessura crustal, utilizando-se o método de interpolação krigagem.

Os valores de profundidade da interface crosta-manto variam entre $34 \mathrm{~km}$ (porção onshore) e $10 \mathrm{~km}$ (porção offshore), sendo apresentados na Figura 5.

A morfologia do Cone do Amazonas é assinalada no mapa de espessura sedimentar (Figura 6), que representa as camadas equivalentes à seqüência rifte, sequiência marinha 1 e sequiência marinha 2. Também destacca-se 0 trend estrutural SW-NE, coincidente com as porções de maiores espessuras, refletindo as feições estruturais do embasamento da bacia.

0 mapa de espessura crustal (Figura 7), considerando a carga sedimentar, demonstra valores entre $34 \mathrm{~km}$ (porção onshore) e $8 \mathrm{~km}$ (porção offshore).

É relevante mencionar que os dados sísmicos não permitiram verificar a geometria e a profundidade da interface crosta-manto. Estes parâmetros foram inferidos pela modelagem gravimétrica $2 \mathrm{D}$.

$\mathrm{Na}$ tentativa de refinar os resultados alcançados em relação à interface crosta-manto, aplicou-se uma técnica de inversão de dados gravimétricos.

\section{INVERSÃO DE DADOS GRAVIMÉTRICOS}

\section{Fundamentos}

Conceitualmente, a determinação da geometria da interface crosta-manto na região do Cone do Amazonas constitui um problema inverso, cuja solução pode ser vinculada ao tratamento matemático dos dados gravimétricos disponíveis.

0 princípio que norteia a resolução do problema inverso em questão é simples (em tese). Fixando-se o contraste de densidade entre a crosta e o manto superior, a morfologia e as profundidades da interface

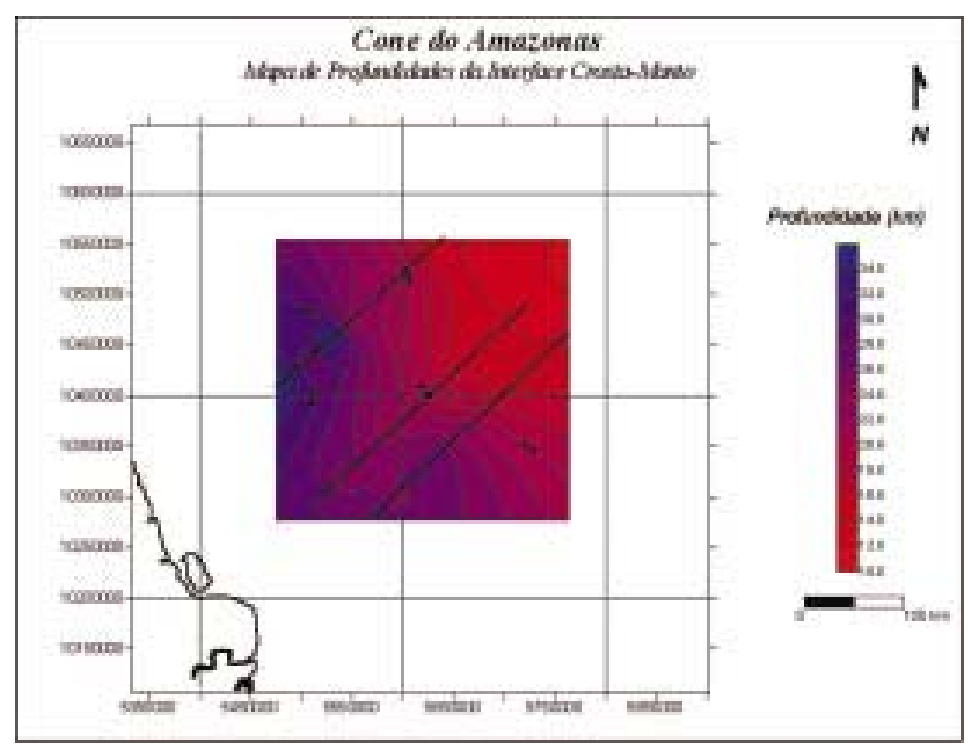

Figura 5 - Mapa de profundidades da interface crosta-manto obtido pela modelagem gravimétrica $2 \mathrm{D}$ Figure 5 - Map of depths to crust-mantle interface obtained by 2D-gravity modelling 


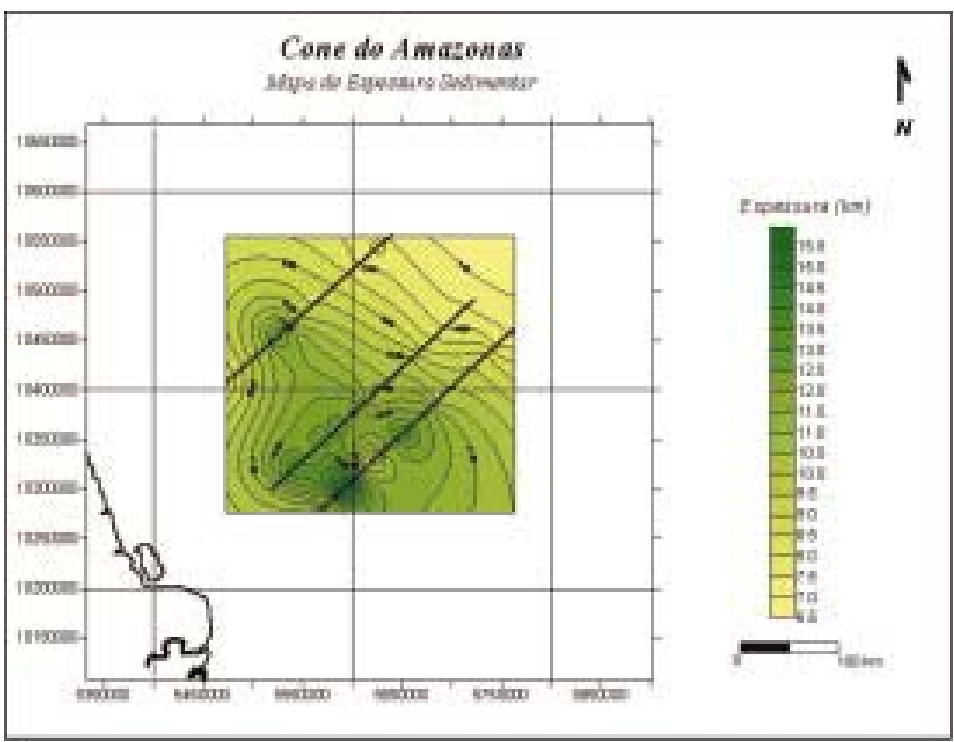

Figura 6 - Mapa de espessura sedimentar (seqüência rifte + seqüência marinha $1+$ seqüência marinha 2) Figure 6 - Map of sedimentary thickness (rift sequence + marine sequence 1 + marine sequence 2)

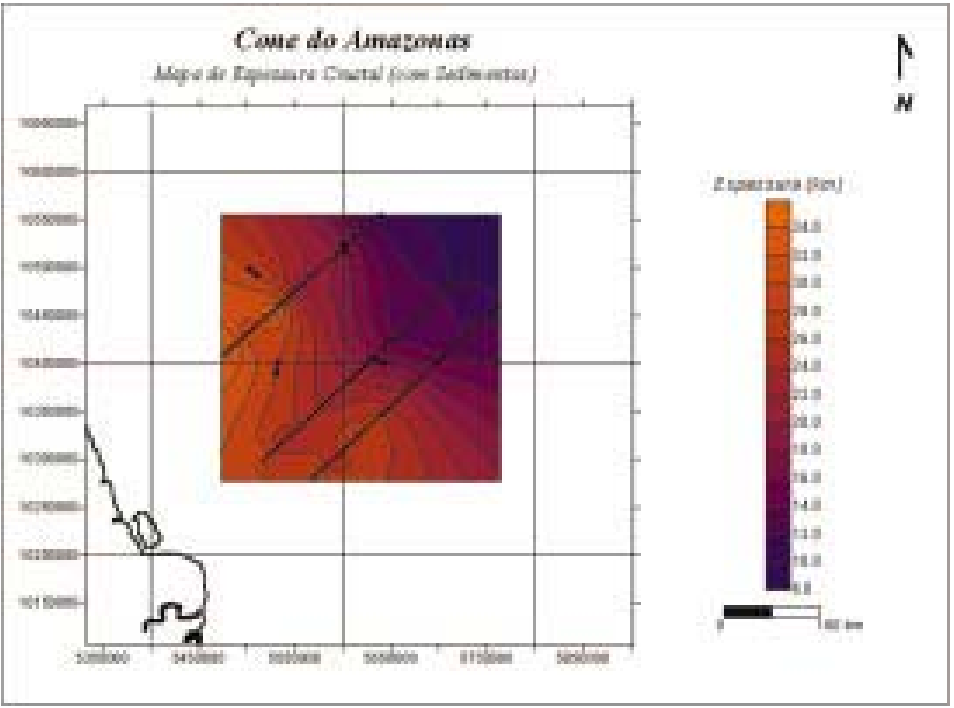

Figura 7 - Mapa de espessura crustal resultante da modelagem gravimétrica Figure 7 - Map of crustal thickness obtained by gravity modelling 
que separa os dois meios podem ser determinadas com o emprego de um algoritmo de inversão não-linear (SCALES; SMITH; TREITEL, 2001).
Utilizou-se um algoritmo de inversão para anomalias gravimétricas (MURTHY; RAO, 1989) com algumas modificações, referentes a corpos bidimensionais, onde a superfície causadora das

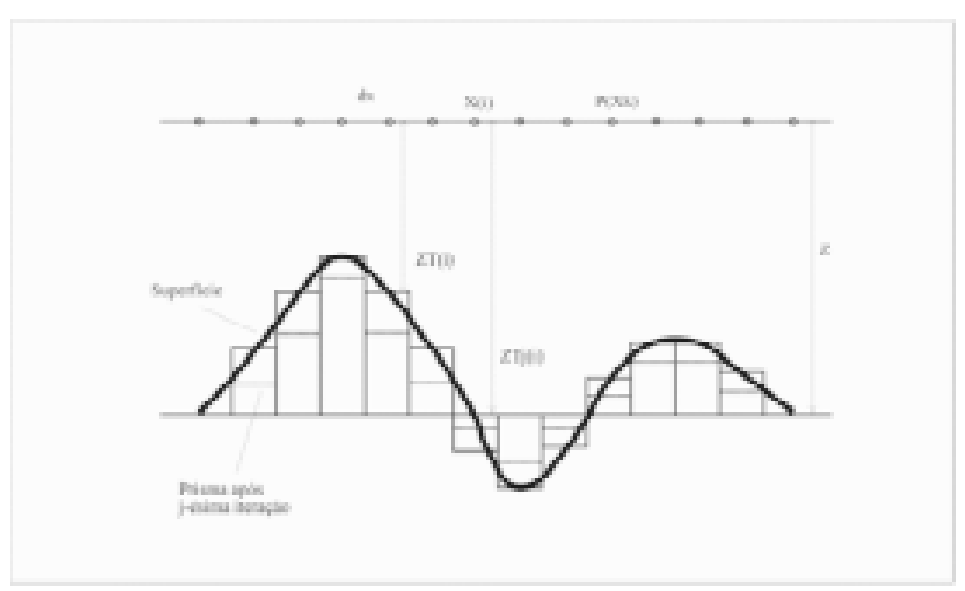

Figura 8 - Superfície modelada por prismas (MURTHY; RAO, 1989)

Figure 8 - Surface represented by prisms (MURTHY; RAO, 1989)

anomalias tem sua profundidade de topo estimada ao longo de um perfil gravimétrico.

A metodologia descrita pelos autores supracitados baseia-se na aproximação desta superfície por um conjunto de prismas justapostos, através de um procedimento computacional iterativo, onde, para cada valor da anomalia gravimétrica $(\mathrm{Dg})$ estima-se a profundidade de topo (ZT) correspondente (Figura 8).

Assim, para um ponto $\mathrm{P}\left(\mathrm{x}_{\mathrm{k}}\right)$ do perfil gravimétrico, a anomalia associada $\Delta \mathrm{g}(\mathrm{k})=\Delta \mathrm{g}\left(\mathrm{x}_{\mathrm{k}}\right)$ é expressa pela equação:

$$
\Delta g(k)=\sum_{i=2}^{N-1}\left[\mathcal{F}_{k}(z)_{Z T(i)}^{Z}+A x_{k}+B\right]
$$

com

$$
\begin{aligned}
& F_{k}(z)=2 G p\left\{z\left[\arctan \left(\left(x_{k}+d x / 2\right) / z\right)-\arctan \left(\left(x_{k}-d x / 2\right) / z\right)\right]+\right. \\
& \left.+0,5\left[\left(x_{k}+d x / 2\right) \ln \left(\left(x_{k}+d x / 2\right)^{2}+z^{2}\right)-\left(x_{k}-d x / 2\right) \ln \left(\left(x_{k}-d x / 2\right)^{2}+z^{2}\right)\right]\right\}
\end{aligned}
$$

onde é e espaçamento entre as estações, $Z T(i)$ é a profundidade do topo da superfície abaixo da i-ésima estação, $r$ representa o contraste de densidade entre as camadas separadas pela superfície-alvo, e $A$ e $B$ os coeficientes da anomalia gravimétrica.

Após a j-ésima iteração, o valor da anomalia calculada pelo modelo prismático $\Delta g_{\text {cal }}(k)$ associada à profundidade $Z T, i$ é fornecida pela equação:

$$
\Delta g_{\text {cal }}(k)=\sum_{i=2}^{N-1}\left[F_{k}(z)_{z T(j)}^{z}+A_{j} x_{k}+B_{j}\right]
$$

Derivando $F_{k}(z)$ em função de $z$, obtém-se:

$\frac{\vartheta F_{k}(z)}{\vartheta z}=-2 G \rho\left[\arctan \left[\frac{x_{k}+d x / 2}{z}\right]-\arctan \left[\frac{\left(x_{k}-d x / 2\right)}{z}\right]\right]$

que é a equação em que se baseia o método de inversão não-linear aqui aplicado.

\section{Metodologia de inversão dos dados gravimétricos}

A metodologia de inversão utilizada é baseada em Murthy e Rao (1989). Inicialmente são calculadas as anomalias de gravidade e as derivadas verticais relativas ao conjunto de prismas, seguidas pela etapa 
de resolução do sistema de equações diferenciais preconizado pela equação (4).

Contudo, o código proposto por Murthy e Rao (1989) foi modificado com o objetivo de se adotar uma variação exponencial das densidades em relação à profundidade. A utilização de um contraste de densidades - variando exponencialmente com a profundidade -, objetiva tornar os resultados da inversão mais realistas, sob o ponto de vista geológico, uma vez que 0 parâmetro densidade não é uniforme em relação à profundidade na crosta terrestre.

Segundo Athy (1930 apud CHAl; HINZE, 1988), o contraste de densidade $\rho_{z}$ de rochas a uma profundidade $\mathrm{z}$ é dado pela equação:

$$
\rho_{z}=\rho_{0} e^{-c z}
$$

onde $r_{o}$ representa o contraste de densidades na superfície e $c$ a constante de decaimento.

Portanto, os seguintes procedimentos foram tomados na inversão não-linear dos dados gravimétricos da região do Cone do Amazonas:

a) filtragem dos perfis gravimétricos, baseada na análise espectral realizada por Oliveira (2003), visando retirar a influência da lâmina d'água e dos sedimentos; b) montagem de três arquivos em formato ASCII contendo quantidade e valores de anomalias em cada perfil, intervalo entre as mesmas, profundidade acima da qual a interface será determinada, limites máximo e mínimo de profundidade da interface, contraste de densidades e fator de decaimento;

c) execução do código proposto por Murthy e Rao (1989), devidamente modificado;

d) geração de três arquivos, em formato ASCII, contendo os valores das anomalias observadas e calculadas e das profundidades da interface.

Os limites máximo e mínimo da profundidade da interface crostamanto foram extraídos dos resultados obtidos na modelagem gravimétrica $2 \mathrm{D}\left(Z \mathrm{ZT}_{\max }=34 \mathrm{~km} ; \mathrm{ZT}_{\min }=8 \mathrm{~km}\right)$.

0 contraste de densidade adotado para a interface foi de $\rho_{0}=$ $0.43 \mathrm{~g} / \mathrm{cm}^{3}$. 0 valor assumido para a constante c foi de 0.0187 (XIA; SPROW, 1995), realizaram uma série de testes numéricos objetivando determinar um valor de $c$ que melhor se aplica à equação (5), na determinaçãa de um modelo de variação de densidades na crosta continental.

0 modelo matemático utilizado na inversão dos dados gravimétricos tem seus resultados expressos nos mapas de profundidade da interface crosta-manto (Figura 9) e de espessura crustal (Figura 10).

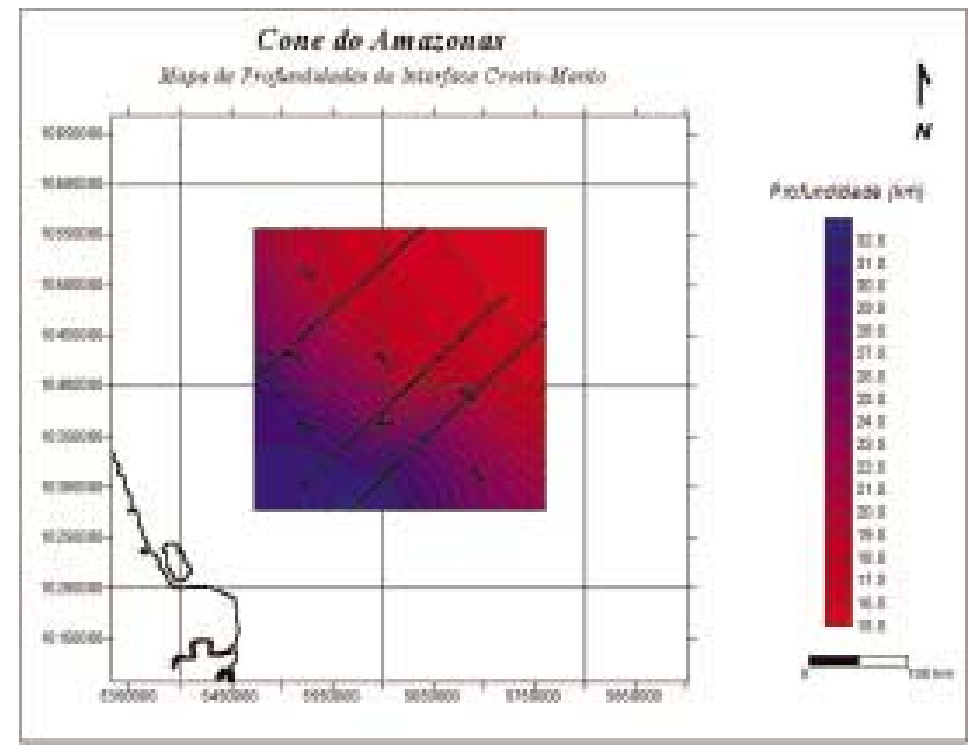

Figura 9 - Mapa de profundidades da interface crosta-manto resultante da inversão dos dados gravimétricos Figure 9- Map of depths to crust-mantle interface obtained by inversion of gravity data 


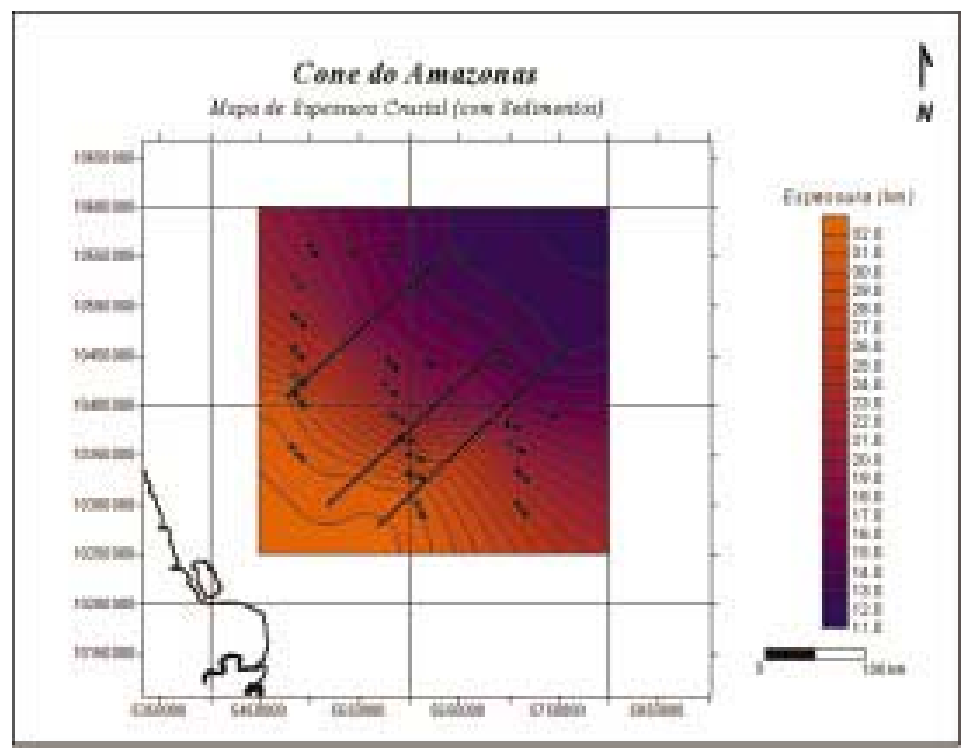

Figura 10 - Mapa de espessura crustal resultante da inversão de dados gravimétricos Figure 10 - Map of crustal thickness obtained by inversion of gravity data

Os valores de profundidade da interface crosta-manto obtidos estão de acordo com os alcançados por experimentos de sísmica de refração conduzidos pela Petrobrás (BRAGA, 1991) na área em questão (maiores que $9 \mathrm{~km}$ e menores que $33 \mathrm{~km}$ ). Outro fato notado é a alteração no padrão dos contornos, em comparação ao mapa da Figura 5, que pode ser justificada pela suavização das profundidades da interface crosta-manto.

0 mapa de espessura crustal incluindo sedimentos apresentou uma correçã̃o nos valores, variando entre o máximo de $32 \mathrm{~km}$ e 0 mínimo de 11 km, além de uma suavização no padrão dos contornos.

\section{DISCUSSÃO}

Na discussão que se segue, são comentados os resultados obtidos pela interpretação de dados gravimétricos na região do Cone do Amazonas, bem como algumas hipóteses que possam ser assumidas como explicações.

Neste trabalho, a delimitação de uma possível crosta transicional na área de estudo foi realizada. Oliveira (2003) já tinha inferido sua suposta existência nas seções sísmicas de reflexão e no estudo dos gradientes vertical e horizontal dos perfis gravimétricos. Outros indicativos de sua presença são anomalias de alta frequiência no perfil GRAV02, que podem ser atribuídas a injeções de material proveniente do manto superior (SPERLE; MELLO; COSTA, 1989), e que podem estar correlacionadas a formação da crosta transicional num estágio inicial de estiramento crustal.

A modelagem gravimétrica $2 \mathrm{D}$ também permitiu a delimitação de diápiros de argila, bem como a estruturação do embasamento da região. Estas feições são consequiência da singular evolução tectônica da região sob um domínio transcorrente, combinada com efeitos decorrentes da expressiva carga sedimentar resultante da deposição do Cone do Amazonas.

Mapas de profundidade da interface crosta-manto e de espessura crustal demonstram o padrão típico assinalado em margens continentais, onde as maiores espessuras encontram-se na porção onshore e as menores espessuras na porção offshore. No mapa de espessura sedimentar observam-se os depocentros da bacia, com espessuras máximas em torno de $15 \mathrm{~km}$, além de destacar a morfologia em leque do Cone do Amazonas.

$\mathrm{Na}$ tentativa de refinar as estimativas de profundidade da interface crosta-manto, um algoritmo de inversão não-linear foi aplicado aos dados gravimétricos, adotando-se um modelo exponencial de distribuição de densidades. Como resultado principal, os valores de profundidade foram corrigidos, mostrando-se coerentes com dados de sísmica de refração (BRAGA, 1991). 
Os resultados aqui apresentados foram utilizados por Oliveira (2003) em estudos tectonofísicos (modelagem numérica e análise flexural), visando determinar as características mecânicas da litosfera na região. Com base nos valores de profundidade do embasamento obtidos pela modelagem $2 D$, aquele autor calculou a rigidez flexural ( $D$ $=1.05 \times 10^{23} \mathrm{~N} \mathrm{~m}$ ) e a espessura elástica efetiva $(\mathrm{Te}=25 \mathrm{~km})$, que corroboram com um modelo de compensação isostática regional para 0 Cone do Amazonas. Este modelo, que parte da premissa de deposição da carga sedimentar sobre uma litosfera termalmente mais fria, explicaria a deformação em regiões adjacentes ao Cone do Amazonas, bem como sua estruturação geológica.

\section{CONCLUSÕES}

A interpretação de dados gravimétricos (modelagem 2D integrada a inversão não-linear) na região do Cone do Amazonas permitiu 0 conhecimento da arquitetura crustal na região. Com base nas etapas executadas, foi possível propor um modelo para a crosta na região, além de determinar a espessura crustal e a profundidade e morfologia da interface crosta-manto.

Cabe ressaltar que a suavidade da interface crosta-manto obtida no processo de inversão aplicado neste estudo é dependente do resultado fornecido pela modelagem 2D. 0 uso de dados de sísmica de reflexão na construção dos modelos de densidade certamente contribuiu para 0 sucesso da aplicação do algoritmo de inversão.

\section{Agradecimentos}

Os autores agradecem ao Projeto Margem Equatorial (Fundação Gorceix/Escola de Minas), pela disponibilidade de computadores e softwares utilizados no decorrer do trabalho, bem como as sugestões e correções do revisor da RBGf. 0 primeiro autor agradece a CAPES, pela concessão da bolsa de mestrado e a ANP, pelo fornecimento dos dados sísmicos e gravimétricos.

\section{REFERÊNCIAS}

ARAÚJO, T. C. M.; THELLEN, F. A estrutura sísmica dos sedimentos na plataforma do Amazonas. Trabalho apresentado no III Congresso Brasileiro de Geofísica, Rio de Janeiro, 1993. v. 2. p. 1237-1241. BRAGA, L. F. S. Isostatic evolution and crustal structures of the Amazon Continental margin determined by admittance analysis and inversion of gravity data. 161p. 1991. Thesis (PhD)-Oregon State University, Oregon, 1991.
BRANDÃO J. A. S. L.; FEIJÓ, F. J. Bacia Foz do Amazonas. Boletim de Geociências da Petrobras, [S.I.], n. 8, p. 91-99, 1994.

CHAl, Y.; HINZE, W. J. Gravity inversion of an interface above wich the density contrast varies exponetially with depth. Geophysics, [S.I.], n. 53, p. 837-845, 1988.

COCHRAN, J. R. Gravity and magnetic investigations in the Guiana Basin, Western Equatorial Atlantic. GSA Bull., [S.I.], n. 84, p. 3249-3268, 1973.

DAMUTH, J. E.; FLOOD, R. D. Morphology, sedimentation processes and growth pattern on the Amazon deep-sea fan. Geo-Marine Letters, [S.I.], n. 3, p. 109-117, 1984.

KUMAR, N. Amazon Cone: morphology, sediments, age and growth pattern. GSA Bull., [S.I.], n. 86, p. 863-878, 1975.

MENDONÇA, C. A.; EL-ROBRINI, M. Potencialidade dos dados de altimetria por satélite nas pesquisas marinhas: ilustração na Margem Equatorial Brasileira. Trabalho apresentado no III Congresso Brasileiro de Geofísica, Rio de Janeiro, 1993. v. 2. p. 1220-1224.

MURTHY, I. V. R.; RAO, S. J. A fortran 77 program for inverting gravity anomalies of two-dimensional basement structures. Computers \& Geosciences, [S.I.], n. 15, p. 1149-1156, 1989.

NESS, G. E. et al. Project EQUANT: a gravity and magnetics study of the Northen Continental Margin of Brazil. Trabalho apresentado no I Congresso Brasileiro de Geofísica, Rio de Janeiro, 1989. v. 2. p. 821824.

OLIVEIRA, L. G. S. Estudo gravimétrico da região do Cone do Amazonas, Bacia da Foz do Amazonas, Margem Equatorial Brasileira. 2003. 127p. Tese (Mestrado)-Departamento de Geologia, Universidade Federal de Ouro Preto, Ouro Preto, 2003.

SCALES, J. A.; SMITH, M. L.; TREITEL, S. Introductory geophysical inverse theory. Colorado: Samizdat Press, 2001.

SHARMA, P. V. Geophysical methods in geology. Amsterdam: Elsevier, 1986.

SPERLE, M.; MELLO, S. L. M.; COSTA, M. P. A. Modelagem gravimétrica e magnética do limite de crostas continental/oceânica no platô do Rio Grande do Norte. Procedimentos do I Congresso Brasileiro de Geofísica, Rio de Janeiro, 1989. v. 2. p. 863-868.

TALWANI, M.; WORZEL, J. L.; LANDISMAN, M. Rapid gravity computations of two-dimensional bodies with application to the mendocino submarine fracture zone. J. Geophys. Res., [S.I.], n. 64, p. 49-59, 1959.

XIA, J.; SPROWL, D. R. Moho depths in Kansas from gravity inversion assuming exponential density contrast. Computers \& Geosciences, [S.I.], v. 21, n. 2, p. 237-244, 1995.

WAHR, P. V. Geodesy and gravity. Colorado: Samizdat Press, 1996. 


\section{NOTAS SOBRE OS AUTORES}

Luiz Gabriel Souza de Oliveira é geólogo pela Universidade de Braślia (2000). Mestre em Ciências da Terra, com ênfase em Geofísica, pela Universidade Federal de Ouro Preto (2003). Atualmente é professor do Departamento de Física e doutorando do PPG-ECRN do Departamento de Geologia, ambos pertencentes à Universidade Federal de Ouro Preto. Suas áreas de interesse envolvem Gravimetria, Geodésia Física e Física de Interiores Planetários.

Marcelo Martins Neto é doutor pela Universidade de Freiburg/Alemanha em 1993, professor do Departamento de Geologia da UFOP, onde coordena atualmente o programa de pós-graduação em Geologia Estrutural e Tectônica. Pesquisador do CNPq desde 1996 vem atuando nas áreas de análise de bacias sedimentares, tectônica \& sedimentação e geologia do petróleo, possuindo relevante produção científica divulgada em veículos nacionais e internacionais de ampla circulação. Coordena projetos em análise de bacias e avaliaçãa de potencial exploratório em parcerias com companhias de petróleo, para as quais também atua como consultor e instrutor em tectônica de bacias, estratigrafia de seqüências e tectônica \& sedimentação.

Maria Sílvia Carvalho Barbosa graduou-se em Engenharia Geológica em 1987, na Universidade Federal de Ouro Preto. Mestre em Geologia Estrutural, em 1990 no Departamento de Geologia (UFOP) e Doutora em Geofísica, em 2000, no Instituto Astronômico e Geofísico (USP). Professora Adjunta da UFOP desde 1991, onde leciona na graduação e na pós-graduação as disciplinas Geofísica, Tectonofíisica e Perfilagem de Poços. Consultora técnica da Fundação Gorceix / Núcleo de Geologia do Petróleo (NUPETRO), desde 2000, nos projetos de Análise de Bacias desenvolvidos para a Petrobrás. Áreas de interesse: métodos potenciais, sísmica, prospecção geofísica, tectonofísica e modelagem matemática. 